\title{
Letter from the Editors-in-Chief
}

\author{
Francesco Basile $\cdot$ Lorenzo Capussotti
}

Published online: 20 July 2010

(C) Springer-Verlag 2010

“Tempori aptari decet" (Seneca, Medea v. 175)

Over the past decades, the official journal of the Italian Society of Surgery (SIC) "Chirurgia Italiana" has accompanied the tremendous progress surgery has made in our country. Nonetheless, the format of the journal in Italian language has hampered the possibility of the authors to have an international recognition of their studies and researches and, thus, to substantially contribute to the surgical literature. Surgical procedures, operative approaches, patient selection and biotechnologies are rapidly evolving forcing the community of surgeons to a rigorous debate and a continuous refinement of standards of care. Nowadays, valuable scientific contributions must conform to widely accepted criteria for study conception and design, data analysis and results reporting. Therefore, the Italian Society of Surgery has decided to make substantial changes to its official journal with the intent of broadening the scope of its journal in terms of content, visibility and readership. Readers will immediately notice the two major changes in the volume that they now hold: a new name "Updates in Surgery" and a new format in English language.

The first issue of "Updates in Surgery" provides a most timely opportunity to express our appreciation to the SIC president, Prof. Enrico De Antoni, and to the Executive Board for their invitation to serve as Editors-in-Chief, entrusting us with the responsibility to provide an high-quality, peerreviewed journal of surgery and related fields.

It is with excitement and purpose that we start our appointment as the new Editors-in-Chief. We pursued this opportunity because

\section{F. Basile $(\square)$}

Surgical Department, Division of General and Oncologic

Surgery, "Vittorio Emanuele, Ferrarotto e S. Bambino"

Hospital, Catania, Italy

e-mail: basilef@live.it

\section{Capussotti}

Surgical Department, Division of Hepato-Bilio-Pancreatic and Digestive Surgery, Mauriziano "Umberto I" Hospital, Torino, Italy e-mail: 1capussotti@gmail.it first and foremost we are committed to give international visibility to the culture of excellence and quality of the Italian surgery. Thus, the highest priority of the new Editorial Board will be to provide high-quality articles (Editorials, Review articles, Original articles, Technical notes and Controversies in surgery) that encompass a broad spectrum of topics with particular regard to innovative surgical approaches and technologies. Yet, we are also aware that regardless of any action we may take, the society and our readers will remain the ultimate judges of the content and value of the journal as reflected by their submissions and service as reviewers.

We are also pleased to announce that we have moved to a more modern online submission system managed by SpringerVerlag. For authors, it will be easier to submit a manuscript. To online submit a manuscript visit the SIC website (http://www.sichirurgia.org/) and click on "Submission" or go to http://www.editorialmanager.com/upis/.

To close, we wish to thank every individual who has participated in preparing this edition of the journal devoting precious time and energy to the process that has generated this volume. We look forward to working with both the Italian and the worldwide surgical community to expand the role of our journal. Thank you all for your support in this important endeavor.

\section{Conflict of interest None.}

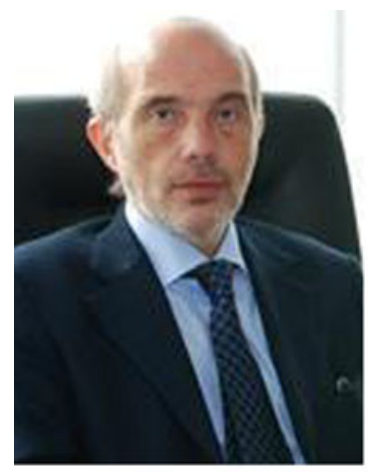

Francesco Basile

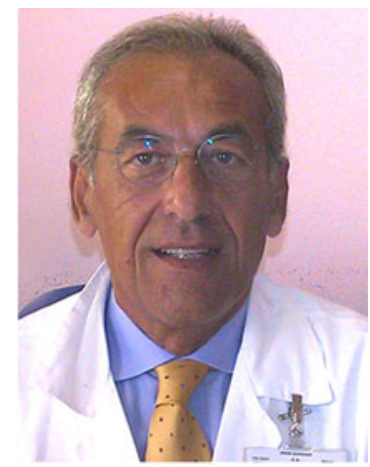

Lorenzo Capussotti 